\title{
Review and prospect of Underwater Target Feature Extraction Based on Active Sonar
}

\author{
Zhong Wang ${ }^{1, a}$, Wei Feng ${ }^{1, b}$ and Xinpeng Wang ${ }^{1, c}$ \\ ${ }^{1}$ Department of Weapon Engineering , Naval University of Engineering , Wuhan 430033 , China \\ adtoubaby@163.com, b476507122@qq.com, ' $1204297054 @ q q . c o m$
}

Keywords: sonar, feature extraction, object recognition, underwater objects.

Abstract. This paper review and prospect the characteristics of active sonar underwater target feature extraction, which is commonly used at home and abroad, and analyzes the main technical difficulties.

\section{Introduction}

In the military application of sonar equipment, mainly used to find, track and identify submarines, mines and other military targets. In order to improve offensive weapons (such as smart torpedoes, submarines) autonomy and effectiveness, the study of military target characteristic is extremely important. In order to be suitable for underwater environment operations, the international carried out intelligent underwater robot research. As the current demand for intelligent sonar equipment is getting higher and higher, and the application of underwater target recognition technology is also more and more, therefore, both in the military or civilian areas, underwater target feature extraction and identification technology will be the future of ships and marine engineering to study one of the main technology. At present, the correct identification of underwater targets is still based on the effective feature extraction based on, so only to extract the characteristics of the other objectives, in order to ensure a more accurate target detection and identification.

The ultimate purpose of the sonar signal processing is to extract the corresponding characteristics of the space and time field, and the operator (or automaton) can interpret the sound waves that produce the sound field according to these characteristics. These explanations include determining the presence or absence of a sound source (or target), its parameters, the dynamic changes, and the nature of what. These analysis processes include: target detection, parameter estimation and positioning, target tracking, target classification and identification and visual display processing and other five functions.

\section{Underwater Target Feature Extraction Using 1D Sonar Data}

Bright Spot of the Structural Characteristics of the Target. Bright spot originally used to describe the concept of a convex smooth surface reflected wave, depending on the fact that the first Fresnel zone. In the case of optics, we can observe such bright spots. The theoretical analysis and experiments have proved that in the case of high frequency (large $k a$ ), any complex target echo is composed of several sub-echo superposition. Each sub-echo can be seen as a wave of a scattering point, the scattering point is a bright spot. It can be a real bright spot, it can be an equivalent bright spot. In this way, any complex target can be equivalent to a combination of several scattered bright spots, each scattered bright spot produces a bright echo, the total echo is the result of these bright echo superposition.

The different bright spots are offset from each other on the acoustic axis, forming a feature along the distance (or two-dimensional space) distribution. Therefore, if the short pulse excitation target, echo can be clearly separated from the bright spot echo, this feature provides the analysis of the bright spot and its characteristics of the basic way. When the incident - reflection azimuth changes, the relative distance between the bright spots and the sound path changes. Therefore, the distribution of bright spots is closely related to the azimuth angle, which leads to various temporal and spatial characteristics of echo. 
Resonance Modal Analysis. In the past 20 years to develop a resonance scattering theory. According to Newton's law, all still objects tend to remain in a resting state, and moving objects tend to maintain their movement. For a structure, when a sinusoidal signal is applied to it, most of the energy is reflected until a certain vibration occurs in the structure, and the object vibrates in all its resonant modes. These resonant modes are contained in the power spectrum of the loaded signal, and the energy associated with these resonant modes is absorbed and stored in the structure, and the remaining energy is reflected.

Typically, the resonant structure of its size, shape and composition of the material related to the structure.The acoustic propagation theory indicates that the velocity of sound propagating in a substance is the square root of the ratio of the modulus of a substance to its density $\rho$, that is, $c=\sqrt{E / \rho}$, and the wave number of the substance is $k=2 \pi f / c$. For a cylindrical object, its radius $a$ is an important physical quantity that reflects the size, and each resonant frequency will provide a data point on the $k a$ field, and a set of such resonant data points will provide a sample description of the distinguishing target. Because the target is always composed of limited species and its size is limited, it is theoretically possible to establish a target resonance mode library for some targets and to compare it with subsequent observations.

Wavelet Transform and Spectrum Analysis. In the field of signal processing, spectrum analysis is one of the most important means of processing. For the sonar one-dimensional echo signal (time series), some parameters of the time domain and frequency domain can be used as the characteristics of the analysis signal. Kundu, etc. in the one-dimensional sonar echo signal classification, the application of the signal feature is: AR model parameter, Fourier power spectrum, wavelet transform parameters.

AR model parameter. In random signal spectral analysis, a time series $x(t)$ is often modeled as a white noise sequence through the output of a digital filter. In general, the filter transfer function $H(z)$ can be written in the form of rational fraction

$$
H(z)=\frac{G}{1-\sum_{i=1}^{P} a_{i} z^{-i}} .
$$

Where the coefficients $a_{i}$ and the gain factor $G$ are model parameters. If $H(z)$ is defined by Eq. 1, this signal model is called the AR model (Autoregressive Model). Signal modeling problem is actually to solve the problem of model parameter estimation. When the sonar signal $x(t)$ is known, the AR model parameters $a_{i}$ and $G$ can be estimated by linear prediction error filtering.

Fourier Power Spectrum. For a given data segment, its short-time Fourier transform parameters are calculated using FFT. Before doing FFT, apply data to each segment. Depending on the situation, you can choose a different window shape. The amplitude of the FFT parameter is the Fourier power spectrum of the data.

Wavelet Transform. In the short-time Fourier transform, the temporal and frequency resolution is fixed. According to the Heisenberg principle, the product of time and frequency resolution can not be greater than $1 / 4 \pi$.If expressed in wavelet, the time resolution can be increased at the expense of the frequency resolution, or vice versa. In the wavelet transform, this concession can result in a high temporal resolution for the high frequency signal and a high frequency resolution for the low frequency signal. The wavelet transform representation is theoretically attractive.

The transformation space of the wavelet transform is defined by some basis functions, and these functions are derived from a base wavelet (obtained by transforming and transforming the base wavelet). For example, $h(t)$ is the base wavelet, $h_{a, \tau}(t)$ is wavelet basis function, then

$$
h_{a, \tau}(t)=\frac{1}{\sqrt{a}} h\left(\frac{t-\tau}{a}\right) .
$$

When $a$ and $\tau$ are continuous, $a$ and $\tau$ can have countless possible values. Therefore, with these basis functions to transform the signal $x(t)$, and then reconstruct the signal from the transform 
domain, the work is not complicated. Interested in how the time domain parameters $a$ and $\tau$ are discretized to construct a true orthogonal wavelet basis function.

\section{Underwater Target Feature Extraction Using 2D Sonar Data (Acoustic Image)}

Due to the development of multi-beam high-frequency sonar and high-resolution imaging sonar, the target feature information extraction technology for underwater target automatic identification system has been developed. The automatic interpretation of sonar images can be divided into three steps: image preprocessing, feature extraction and target recognition, as shown in Fig.1.

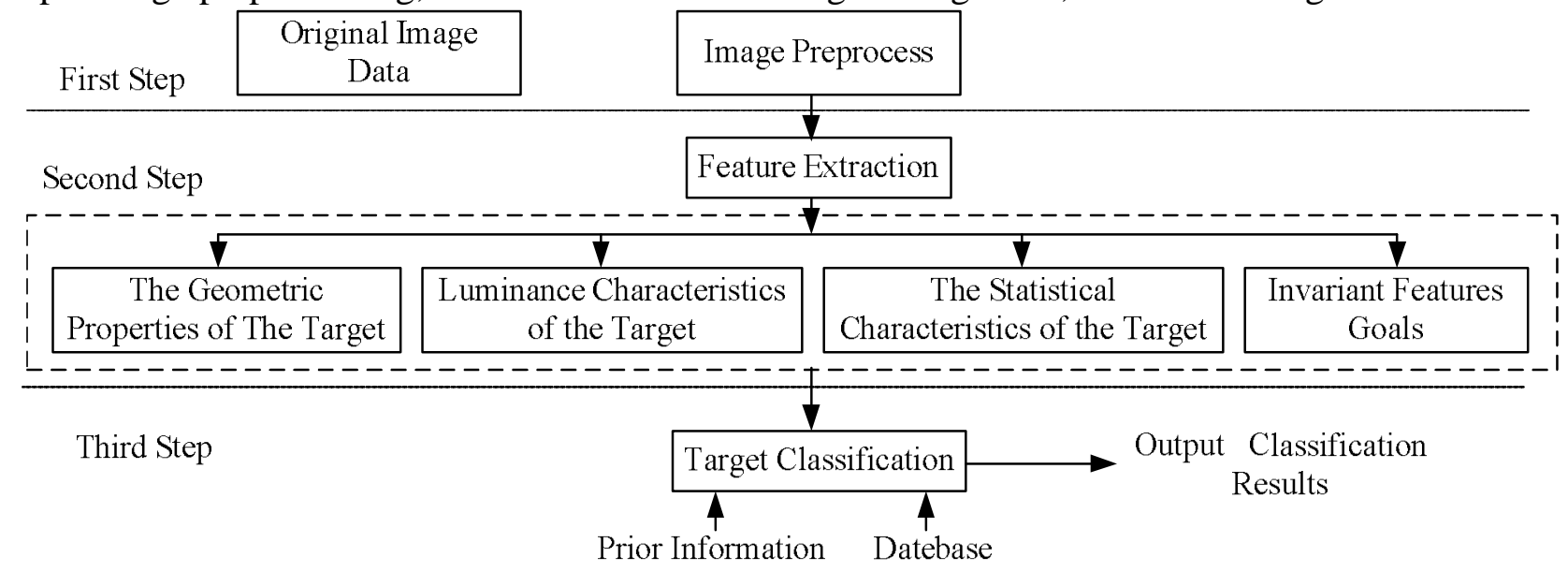

Fig. 1. Automatic Interpretation System sonar image information processing flow chart

Image preprocessing. The image preprocessing phase includes filtering the original image and image segmentation. First of all, the median filter method to filter the original image, the general window with $3 \times 3$ or $5 \times 5$ median filter to eliminate part of the noise. The background level is estimated using a $21 \times 21$ median filter.

For image segmentation, the image is usually divided into three types of regions: the target area, the shadow area, and the background area. In the Cartesian coordinate system, $(x, y)$ represents the position of the pixel, and $f(x, y)$ represents the gray level of the pixel at the $(x, y)$ point. Image segmentation is the pixel is classified as one of the three regions. The commonly used method is a double threshold method, that is, the two thresholds $T_{u}$ and $T_{i}$ are selected, and if $f(x, y) \geq T_{u}$, the pixel $(x, y)$ is classified as the target bright spot; If $f(x, y) \leq T_{i}$, the pixels $(x, y)$ are classified as shaded areas; other pixels are classified as background area. The threshold values $T_{u}$ and $T_{i}$ may be taken as fixed values or may be selected adaptively. If there are multiple targets exist, you can split a number of target areas and shadow areas, with different markers to show the difference.

Feature extraction technology. When the sound waves are emitted, they are scattered by sea objects or objects in the water. Objects in the water will block part of the sound waves so that they can not reach the sea, so the sea will produce the so-called sound shadow area, as shown in Fig.2. Fig.2 shows the geometric schematic characteristics of the echo data, including the intensity, size, shape, size of the sound image, and the relative position of the target.

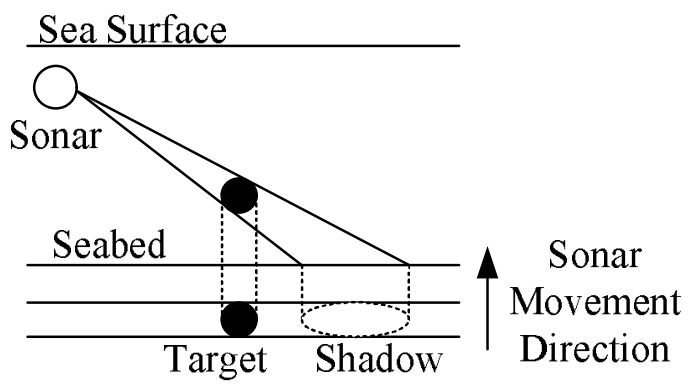

Fig. 2. Geometric Schematic of Recursive Sonar Recorder 


\section{On invariant feature extraction}

When there is a relative position change between the target and the sensor, the observed target shape, size, intensity, etc. will vary. In fact, even if the sonar and the target are still, in different sonar scanning process will also produce changes in the shape of the target. Therefore, for an automatic target recognition system, it should be the same as the human visual system, when the target shape changes can still correctly identify the target, which needs to extract the invariant characteristics of the target.

\section{Difficult Problems in Underwater Target Feature Extraction}

An automatic target recognition system for the real world is currently facing the main problem remains:

1. The amount of raw data is large. To ensure that the automatic identification is successful, the sonar device should have a higher resolution so that the information to be processed has sufficient information. The raw data is limited by the sonar device.

2. Should be invariant. When there is a relative position change between the target and the sensor, the system is required to be able to correctly identify the target.

3. The quality of the data obtained is poor. Environmental interference and sensor error, background noise, etc. to make the data pollution.

4. Real-time. The system is required to identify the target in real time, requiring the system to have very fast preprocessing, feature extraction and recognition speed, and effective signal processing.

\section{Conclusions}

Most of the underwater target feature extraction techniques mentioned in this paper are aimed at underwater targets in close range, where the sonar equipment used is mostly high frequency, high resolution sonar, the role of distance within $200 \mathrm{~m}$. Although the study of these underwater target feature extraction techniques and target recognition methods based on these features, has obtained some experimental (or simulation) results, but these technologies are based on the sonar signal automatically explain the system from the practical there is still some distance, there are still many problems to be solved.

\section{References}

[1] Kundu, A., Chen, G. C., \& Persons, C. E: Transient sonar signal classification using hidden markov models and neural nets. IEEE Journal of Oceanic Engineering, Vol. 19 (1994), p. 87-99.

[2] Hemminger, T. L., \& Pao, Y. H: Detection and classification of underwater acoustic transients using neural networks. IEEE Transactions on Neural Networks, Vol. 5 (1994), p. 712-8.

[3] CHEN. (2010). A method for detecting target echo in reverberation based on fractional fourier transform. Chinese Journal of Acoustics, 40(4), 401-412.

[4] Rashtchi, V., \& Nourazar, M: Fpga implementation of a real-time weak signal detector using a duffing oscillator. Circuits Systems \& Signal Processing, Vol. 34(2015), p. 1-19.

[5] Kryjak, T., Komorkiewicz, M., \& Gorgon, M: FPGA implementation of real-time head-shoulder detection using local binary patterns, SVM and foreground object detection. Design and Architectures for Signal and Image Processing Vol.241 (2012) , p.1-8.

[6] Tang, Q., Liu, B., Chen, Y., Zhou, X., \& Ding, J: Application of lvq neural network combined with the genetic algorithm in acoustic seafloor classification. Chinese Journal of Geophysics, Vol. 50(2007), p. 291-298. 